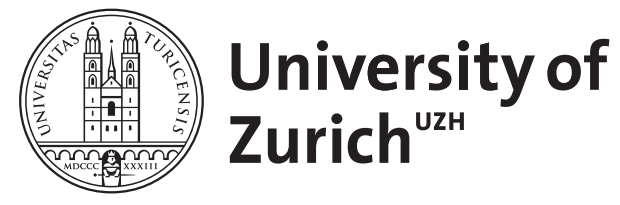
Archive

University of Zurich

University Library

Strickhofstrasse 39

CH-8057 Zurich

www.zora.uzh.ch

Year: 1996

Erwägungen zur Redaktion der Meerwundererzählung (Exodus 13,17-14,31)

Krüger, Thomas

DOI: https://doi.org/10.1515/zatw.1996.108.4.519

Posted at the Zurich Open Repository and Archive, University of Zurich

ZORA URL: https://doi.org/10.5167/uzh-2053

Journal Article

Published Version

Originally published at:

Krüger, Thomas (1996). Erwägungen zur Redaktion der Meerwundererzählung (Exodus 13,17-14,31).

Zeitschrift für die alttestamentliche Wissenschaft, 108(4):519-533.

DOI: https://doi.org/10.1515/zatw.1996.108.4.519 


\title{
Erwägungen zur Redaktion der Meerwundererzählung (Exodus 13,17-14,31)
}

\author{
Von Thomas Krüger
}

(Universität Zürich, Theologisches Seminar, Kirchgasse 9, CH-8001 Zürich)

Die Erzählung vom Untergang der Ägypter im Schilfmeer in Ex 13,17-14,31 (im folgenden kurz: Ex 13+14) bietet eine durchaus kohärente Darstellung eines (bei Bereitschaft zur Imagination nicht-alltäglicher Vorgänge) anschaulich vorstellbaren Ereignisablaufs. ${ }^{1}$ Bei näherem Zusehen läßt der Text jedoch noch deutliche Spuren eines mehrstufigen Wachstums- und Kompositionsprozesses erkennen, für den - im Rahmen umfassenderer literaturgeschichtlicher Hypothesen zu GenDtn (+ Jos-II Reg) ${ }^{2}$ - unterschiedliche Rekonstruktionen vorgeschlagen worden sind. ${ }^{3}$

Die klassische "Quellenscheidung ${ }^{4}{ }^{4}$ verteilte den Textbestand auf die drei Quellen Jahwist (J), Elohist (E) und Priesterschrift $(\mathrm{P})$ und nahm an, daß sich die Tätigkeit der Redaktoren im wesentlichen auf das mehr oder weniger geschickte - Zusammenfügen vorgegebenen Textmaterials $(\mathrm{J}+\mathrm{E}$ bzw. $\mathrm{P}+\mathrm{JE})$ beschränkt habe. Demgegenüber rechnen neuere redaktionsgeschichtliche Erklärungsmodelle nur noch mit zwei im Text verarbeiteten Quellen, die z. T. noch Spuren literarischen Wachstums erkennen lassen, sowie mehr oder weniger umfangreichen weiteren Zusätzen im Zuge und im Gefolge ihrer redaktionellen Verbindung. ${ }^{5}$

1 Vgl. etwa J.-L. Ska, Le passage dans la mer, AnBib 109 (1986).

2 Vgl. zur neueren Diskussion etwa L. Schmidt, Zur Entstehung des Pentateuch, VF 40 (1995), 3-28; E. Otto, Kritik der Pentateuchkomposition, ThR 60 (1995), 163-191; H. Utzschneider, Die Renaissance der alttestamentlichen Literaturwissenschaft und das Buch Exodus, ZAW 106 (1994), 197-223.

3 Vgl. M. Vervenne, The Question of "Deuteronomic " Elements in Genesis to Numbers, in: Studies in Deuteronomy in Honour of C. J. Labuschagne, 1994, 243-268: $254 \mathrm{ff}$. (Lit.). - Aus Raumgründen muß die Diskussion mit der Sekundärliteratur im folgenden auf ein Minimum beschränkt werden.

4 M. Noth, Überlieferungsgeschichte des Pentateuch, 1948; ders., Das zweite Buch Mose - Exodus, ATD 5, 1958; A. F. Campbell/M. A. O'Brien, Sources of the Pentateuch, 1993, 238 ff.; F. Kohata, Jahwist und Priesterschrift in Exodus 3-14, BZAW 166, 1986; dies., Die Endredaktion $\left(\mathrm{R}^{\mathrm{P}}\right)$ der Meerwundererzählung, AJBI 14 (1988), 10-37. Vgl. Vervenne, Question, 257 mit Anm. 44.

5 P. Weimar, Die Meerwundererzählung, ÄAT 9, 1985; C. Levin, Der Jahwist, FRLANT 157, 1993; H. Lamberty-Zielinski, Das "Schilfmeer", BBB 78, 1993. Vgl. Vervenne, Question, 257 f. mit Anm. 48. 
Daneben wurde auch vorgeschlagen, auf die Annahme verschiedener "Quellen « in Ex 13+14 ganz zu verzichten und den traditionell der Priesterschrift zugeschriebenen Textanteil als Grundlage einer redaktionellen Bearbeitung ${ }^{6}$ bzw. als redaktionelle Bearbeitung einer vorgegebenen Grundlage $^{7} \mathrm{zu}$ interpretieren.

Mit den folgenden Überlegungen möchte ich ein Erklärungsmodell zur Diskussion stellen, das in Ex 13+14 neben zwei Quellen mit einer substantiellen Redaktion rechnet, die sich nicht nur um den Ausgleich von Spannungen zwischen den verschiedenen ihr vorliegenden Darstellungen des Meerwunders bemüht, sondern dieses zugleich auch in umfassendere Horizonte literarischer Komposition und theologischer Interpretation einbindet. Dabei geht es mir weniger um die interne literarische (Un-)Einheitlichkeit dieser drei Textkomponenten als um ihre relative Abgrenzung und Abfolge in der Textgeschichte. ${ }^{8}$

\section{I}

Hinsichtlich der Abgrenzung des priester(schrift)lichen Textanteils 9 in Ex 13+14 besteht in der Forschung weitgehende Übereinstimmung. ${ }^{10}$ Im Kontext schließt er bruchlos an $12,40 \mathrm{f}$. ( $\mathrm{P}^{\mathrm{G}}$ ?) bzw. 12,51 ( $\mathrm{P}^{\mathrm{S}}$ ?) an und findet seine Fortsetzung in 15, $* 22-27 ; 16, * 1 \mathrm{ff} .{ }^{11}$ Selbst Erhard Blum gesteht "der priesterlichen Komponente in Ex 14 « ein hohes Maß an "Geschlossenheit und Eigenständigkeit " zu. ${ }^{12}$ Angesichts der "Leerstelle" vor 14,15a - "von einem Schreien Moses zu Gott ist davor nicht die Rede" - rechnet Blum für Ex 13+14 mit der (unvollständigen) "Aufnahme eines vorgegebenen, bereits formulierten Textes " durch seine

6 H.-C. Schmitt, "Priesterliches" und "prophetisches" Geschichtsverständnis in der Meerwundererzählung Ex 13,17-14,31, in: Textgemäß, FS E. Würthwein, 1979, 139155 .

$7 \mathrm{M}$. Vervenne, The "P«-Tradition in the Pentateuch: Document and/or Redaction?, in: C. Brekelmans/J. Lust (eds.), Pentateuchal and Deuteronomistic Studies, BEThL 94, 1990, 67-90; J. Van Seters, The Life of Moses, Contributions to Biblical Exegesis and Theology 10, 1994, 131 ff.; ähnlich E. Blum, Studien zur Komposition des Pentateuch, BZAW 189, 1990, $256 \mathrm{ff}$. Vgl. Vervenne, Question, 258 mit Anm. 48 und 50.

8 Vgl. zu den folgenden Ausführungen jeweils die Übersetzung im Anhang.

9 14,1.2a3.4.8a* (ohne mlk mṣrym). 10abß.15.16* (ohne hrm 't mṭ w-). 17.18.21a $\alpha^{1}$.b. 22.23.26.27a $\alpha^{1} .28 \mathrm{a} .29$.

10 Vgl. zur unterschiedlichen Abgrenzung im einzelnen die bei Vervenne, Question $257 \mathrm{f}$., referierten Vorschläge. Zur weiteren Diskussion sowie zur gelegentlichen Bestreitung eines P-Anteils in Ex $13+14$ s. die Hinweise bei Blum, Studien, $258 \mathrm{f}$., und Schmitt, Geschichtsverständnis, 144.

11 Vgl. N. Lohfink, Studien zum Pentateuch, SBAB 4, 1988, 110.231 (zu 13,20 s. u.); E. Ruprecht, Stellung und Bedeutung der Erzählung vom Mannawunder (Ex 16) im Aufbau der Priesterschrift, ZAW 86 (1974), 269-307; Blum, Studien, 144 ff.

12 Blum, Studien, 260. 
'priesterliche Kompositionsschicht $\triangleleft$ Damit kommt er für Ex 13+14 der klassischen 'Quellenscheidung, recht nahe. ${ }^{13}$ Für eine Quelle, in der 14,15a unmittelbar an 14,10* anschließt, läßt sich die Leerstelle vor V. 15a freilich durchaus plausibel erklären, wenn man davon ausgeht, $\mathrm{da} ß$ in 14,15a "Mose als der Sprecher der sschreienden Israeliten angeredet wird « ${ }^{14}$ bzw. daß er in 14,10 schlicht mit den übrigen Israeliten "geschrieen « hat.

Die priesterschriftliche Meerwundererzählung läßt eine klare Konzeption erkennen: Mit der wiederholten Abfolge von Ankündigung und Ausführung in $14, * 1-10 . * 15-23$ und *26-29 demonstriert sie die souveräne Lenkung des Geschehens durch Jahwe. Während Jahwe auf die Ägypter durch die "Verhärtung" des Herzens Pharaos einwirkt (V. 4.8*.17), lenkt er Israel durch Anweisungen, die Mose dem Volk übermittelt (V. $1 \mathrm{f} .15 \mathrm{f}$.). Dabei ist allem Anschein nach nur Mose über die weiteren Pläne Jahwes zum voraus im Bilde ${ }^{15}$, und auch er jeweils nur über die nächsten Schritte (V. 3 f.17 f.). Aus den Israeliten herausgehoben ist Mose aber vor allem dadurch, daß er das Meerwunder auf Geheiß Jahwes direkt selbst bewirkt (V. 16.21*). Ziel des Ganzen ist die "Verherrlichung " Jahwes an Pharao und seinem Heer und seine "Erkenntnis" durch die Ägypter (V. 4.18). Allein diesem Zweck dient das Meerwunder - nicht etwa der "Rettung" Israels, das im souveränen Kalkül Jahwes zu keinem Zeitpunkt des Geschehens ernsthaft bedroht war. ${ }^{16}$

Das unterstreicht der Einsatz der priesterschriftlichen Meerwundererzählung in 14,1 f.: Wenn Israel hier zur "Umkehr " $(\check{s} w b)$ an den Sirbonischen See ${ }^{17}$ aufgefordert wird, scheint vorausgesetzt zu sein, daß es auf seinem Zug von Ägypten nach Kanaan auf der von Pelusium über Raphia nach Gaza führenden Küstenstraße diese Gegend bereits passiert

13 Ebd. Anders als Weimar sieht Blum »keine Möglichkeit, die vorgegebene Überlieferung genau abzugrenzen und zu bestimmen " (ebd. Anm. 119). - Blums These, daß "die vorausgehenden priesterlichen Stücke [...] sich nicht zu einem selbsttragenden Werk [fügen]" (a.a. O. 260), kann hier nicht im einzelnen diskutiert werden. Vgl. dazu etwa L. Schmidt, Studien zur Priesterschrift, BZAW 214, 1993.

14 So u. a. Noth, Exodus, 90, dagegen Blum, Studien, 260 Anm. 118.

15 In Anbetracht dieses "Wissensvorsprungs « wird die Rüge an Mose in 14,15 verständlich: Indem er mit den Israeliten zu Jahwe geschrieen hat, ist er seiner Anführer-Funktion nicht gerecht geworden.

16 Als "Rettungstat " Jahwes konnte das Meerwunder allenfalls den Israeliten erscheinen, die nicht wie Mose im vorhinein über die Absichten Jahwes im Bilde waren. In diesem Sinne ließ sich die jahwistische Version des Meerwunders (vgl. 14,13.30!) aus der Perspektive von $P$ (als Neuschreibung) erklären und kritisch relativieren.

17 Zum Problem der Lokalisierung der in 14,2 genannten Ortschaften vgl. H. Donner, Geschichte des Volkes Israel und seiner Nachbarn in Grundzügen I, GAT 4/1, ${ }^{2} 1995$, 109; Lamberty-Zielinski, Das "Schilfmeer", 128 Anm. 27. 
hat. ${ }^{18}$ Das folgende Meerwunder ist demnach nicht etwa als konstitutiver Bestandteil der Befreiung Israels aus Ägypten zu verstehen, deren Vollzug in 12,40 f. (51) bereits konstatiert wurde. Es wird vielmehr durch die provokative $(14,3)$ "Umkehr " Israels und die "Verhärtung " des Herzens Pharaos von Jahwe planvoll »inszeniert «, um ihm Gelegenheit zu geben, sich "an Pharao und an seinem ganzen Heer " zu "verherrlichen" und sich den "Ägyptern« zu erkennen zu geben $(14,4)$.

Die. Rekonstruktion einer "jahwistischen " Quelle ${ }^{19}$ im nicht-priesterschriftlichen Textbestand von Ex 13+14 ist in der Forschung weit stärker umstritten als die Abgrenzung des priesterschriftlichen Textanteils. ${ }^{20}$ Wichtigster Anhaltspunkt ist neben terminologischen Varianten (»der König von Ägypten " neben "Pharao", 'lhym neben yhwh), Doppelungen $(14,6 / 7.19 \mathrm{a} / \mathrm{b} .30 / 31)$ und Unklarheiten im Geschehensablauf (bes. in 14,19f.) die Beobachtung, daß der nicht-priesterschriftliche Textbestand in Ex 13+14 Erzählungszüge enthält, die im Widerspruch zur priesterschriftlichen Meerwundererzählung auf eine Version des Meerwunders hindeuten, in der dieses nicht nur (1) der Rettung Israels diente (vgl. 14,13.30), sondern sich v. a. auch (2) nicht - wie in P allem Anschein nach vorausgesetzt - am hellichten Tage abspielte, sondern in der Dunkelheit der Nacht ${ }^{21}$, wobei (3) die Israeliten sich keineswegs wie beim priesterschriftlichen Durchzug durch das Meer - fortbewegten, sondern an ihrem Platz verharrten. ${ }^{22}$

18 Das spricht $\mathrm{m}$. E. gegen eine Zuweisung von $13,20 \mathrm{zu}$ P.

$1913,20.21 \mathrm{a \alpha}$ " (ohne ywmm und lnhtm hdrk); 14,5a.6.9a $\alpha^{2} .10 \mathrm{ba} .13-14.19 \mathrm{~b} 20 \mathrm{a} \alpha \beta^{*}$

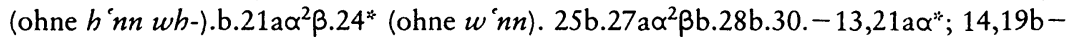

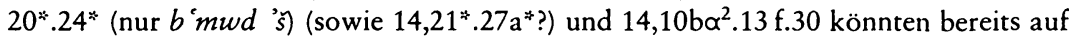
vor-priesterschriftliche Bearbeitung(en) zurückgehen (vgl. E. A. Knauf, Midian, ADPV, 1988, $130 \mathrm{ff}$.), die möglicherweise mit der Erweiterung von Ex *1-14 (15) um Ex *19ff. zusammenhängen.

20 Vgl. die bei Vervenne, Question, 257f. referierten Vorschläge. Die hier vertretene Abgrenzung stimmt im wesentlichen z. B. mit den Rekonstruktionen von Noth und Levin $\left(\mathrm{J}^{\mathrm{Q}}+\mathrm{J}^{\mathrm{R}}\right)$ überein.

21 Vgl. die Zeitangaben in 14,20 f.24.27; zu dem in 14,13 angekündigten "Sehen "Israels kommt es erst nach Anbruch des Morgens: 14,30.

22 14,13: "bleibt stehen! - 14: "ihr werdet euch still verhalten" - 19: "die Wolkensäule ... stellte sich hinter sie«. - Blum, Studien, 257 Anm. 96, registriert diesen »überlieferungsgeschichtlichen [?] Befund «, meint aber: "es [wäre] gut zu verstehen, wenn die Komposition bei diesem zentralen Vorgang eine unnötige Doppelaussage vermied ... Auch die Aufforderung von 14,13 f. ... braucht man nicht in dem Sinne zu pressen, daß die Israeliten sich die ganze Zeit nicht von der Stelle rühren durften. Umgekehrt müßte man nach dem erzählerischen Sinn der aufwendigen Trockenlegung des Meeres obne Durchzug fragen«. Dieser läßt sich jedoch - nach Ausscheidung von 14,25a - 
Verbindet man die so rekonstruierte "jahwistische «"23 Version der Meerwundererzählung mit Ex 12,29-39(*), wird zudem deutlich, daß die Rettung am Meer hier - anders als bei $\mathrm{P}$ - ein konstitutives Element des Auszugs aus Ägypten ist. Aus 12,31 f.(") geht nämlich hervor, daß Pharao die Israeliten zum »Gottesdienst « in der Wüste entlassen hat, wie sie es ab Kap. 5 immer wieder verlangt hatten: »Geht, dient Jahwe, wie ihr verlangt habt! « Dabei rechnet er allem Anschein nach mit ihrer Rückkehr. Erst in 14,5a wird es für Pharao klar (und für die Leser des ExodusBuches eindeutig), daß die Israeliten ihren »Urlaub « zur Flucht genutzt haben. Insofern ist 14,5a keineswegs »eine nach allem bisher Erzählten höchst überraschende Aussage «24, sondern konstitutiver Bestandteil der "jahwistischen « Version des Auszugs aus Ägypten in Ex 1-14. ${ }^{25}$

$\mathrm{Da}$ es sich hierbei um eine (bzw. die) vor-priesterschriftliche Version des Exodus handelt, läßt sich aus dem vorliegenden Text der Meerwundererzählung nicht zwingend begründen. Es scheint jedoch mindestens möglich, die priesterschriftliche Meerwunder-Erzählung als korrigierende Neuschreibung der rekonstruierten »jahwistischen " Quelle zu verstehen. Dabei wäre dann insbesondere auch Mose aufgewertet worden, dem in der "J «-Version allein die Rolle zufiel, Israel zum Vertrauen auf die Hilfe Jahwes zu ermutigen, während Jahwe selbst das Volk (in einer Wolkensäule) anführt und (in einer Feuersäule) für es streitet.

\section{III}

Der nach Ausscheidung von $P$ und " $J$ "verbleibende Textbestand in Ex $13+14^{26}$ läßt sich nun verstehen als Niederschlag redaktioneller Ar-

unschwer erkennen: Wäre das Meer nicht von Jahwe trockengelegt worden, wären die Ägypter bei ihrer panischen Flucht kaum in das Bett des Meeres geraten und von diesem bei seiner Rückkehr überflutet worden. Auch die Bemerkung 14,27b Jahwe habe die Ägypter "ins Meer geschüttelt $\left(n^{\prime} r\right)$ ", paßt besser zur rekonstruierten "J"Version des Meerwunders als zur Durchzugs-Version von $P$ (und R). Entsprechendes gilt für 15,1.21 ( $r m b)$ und 4 (yrh), während 15,8 und 16b (vgl. Jos 3 f.) die Perspektive von $\mathrm{P}$ (bzw. R) bereits vorbereiten oder voraussetzen.

23 Die Frage, ob und wie weit die "jahwistische" Quelle von Ex 13+14 literarisch über Ex "1-14 hinausreichte, kann und muß hier offen bleiben - deshalb die Anführungszeichen.

24 So Noth, Exodus, 88.

25 Vgl. Van Seters, The Life of Moses, 130. Levin, Jahwist, $341 \mathrm{ff}$., interpretiert 14,5b als nachträglichen "Ausgleich " der Meerwundererzählung mit dem von ihm als nachjahwistisch eingestuften Plagenzyklus.

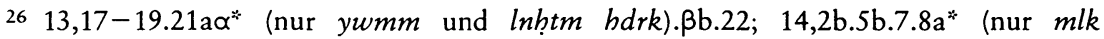

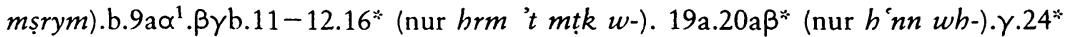
(nur $w^{\prime} n n$ ). 25a.31. - Vgl. zur abweichenden Abgrenzung der nach-priesterschriftlichen Textanteile bei Weimar, Levin und Lamberty-Zielinski das Referat bei Vervenne, Question, 258. 
beit ( $R$ ), die (1) die beiden vorgegebenen Quellen der Meerwundererzählung miteinander verbindet und zwischen ihnen bestehende Spannungen auszugleichen versucht, (2) aus dem vorgegebenen Quellenmaterial eine neue theologische Konzeption des Meerwunders entwickelt und dieses zugleich (3) in weiträumigere literarische und konzeptionelle Horizonte einordnet. Es ist durchaus vorstellbar, daß diese redaktionelle Arbeit in mehreren Stufen durchgeführt wurde. Hier soll es jedoch nur darum gehen, zu zeigen, daß der zur Diskussion stehende Textanteil in Ex $13+14$ im Horizont einer Kombination von " J " und P plausibel interpretiert werden kann.

(1) 13,17 f. bereitet die Verbindung von "J « und P mit ihren differierenden Lokalisierungen des Meerwunders vor (und schließt durch das Stichwort "Schilfmeer " möglicherweise noch eine dritte, in 15,1-21 erhaltene Version des Meerwunders daran an, vgl. 15,4.22). Während nämlich die Priesterschrift das Meerwunder am Sirbonischen See lokalisiert $(14,2)$, deutet die "jahwistische" Abfolge der Itinerar-Notizen in 12,37 (von Ramses nach Sukkot) und 13,20 (von Sukkot nach Etam = "am Meer «: 14,9), die jeweils Tagesmärsche im Auge zu haben scheint, eher auf einen der Seen auf der Landenge zwischen Suez und Port Said als Schauplatz des Meerwunders in der "jahwistischen " Quelle hin. ${ }^{27}$ Diese Divergenz wird in 13,20 ff. dadurch beseitigt, daß die Israeliten zunächst (gemäß » J) von Sukkot nach Etam ziehen $(13,20)$, dort aber dann (nach P) den Befehl zur "Umkehr " nach Pi-Hachirot erhalten $(14,2)$. Der vorliegende Text lokalisiert das Meerwunder dementsprechend (mit P) am Sirbonischen See $\left(14,9 b^{28}\right)$. Der aus dieser redaktionellen Kombination der Quellen resultierende "Umweg " wird in 13,17 f. vorweg begründet. ${ }^{29}$

Eine weitere gravierende Divergenz zwischen den beiden $\mathrm{R}$ vorgegebenen Meerwunder-Versionen besteht darin, daß der Durchzug durch das Meer nach P die Möglichkeit visueller Orientierung für Israeliten wie Ägypter erfordert (und deshalb allem Anschein nach bei Tage erfolgt), während das Meerwunder sich bei "J " in der Nacht abspielt und sein Ergebnis den Israeliten erst am nächsten Morgen sichtbar wird. Unter der Voraussetzung, daß die vorhandenen Quellen in ihrem Wortlaut vollständig bewahrt werden sollten, stellte sich $\mathrm{R}$ damit die Aufgabe, eine Szenerie zu entwickeln, die zunächst einen nächtlichen Zug der Israeliten

27 Vgl. Donner, Geschichte, 108.

28 14,9b wird meist zu P gerechnet (vgl. Schmidt, Studien 19 ff.). Syntaktisch ließe er sich auch mit V. $8 \mathrm{a}^{*}$ oder V. $9 \mathrm{a}^{1}$ verbinden (wenn man die Präposition $l$ im Sinne von "bis nach « versteht).

29 Schon die komplizierte syntaktische Struktur von 13,17 f. mit mehrfachen Rückblenden und Vorblicken (sowie Einblicken in die Überlegungen Gottes) spricht m. E. gegen die Annahme, hier seien Teile einer dritten erzählenden Quelle (E) enthalten; die Verse haben eher den Charaker einer kommentierenden Erläuterung als den einer Erzählung. 
durch und der Ägypter in das Meer ermöglichte, den Untergang der Ägypter im Meer dann aber so darstellen konnte, daß er den Israeliten erst am folgenden Tag erkennbar wurde. Dieses Problem konnte $\mathrm{R}$ mit Hilfe der "Wolkensäule« $\left(13,21^{*}\right)$ und der "Feuersäule « $(14,24 *)$ aus "J» lösen. ${ }^{30}$

$13,21 \mathrm{f}$. funktioniert die "Feuersäule " aus $14,24 *$ zu einer ständigen nächtlichen Lichtquelle für Israel um. Damit wird nicht nur nachträglich die Möglichkeit eines nächtlichen Auszugs der Israeliten aus Ägypten erklärt (vgl. 12,29 ff.) sowie auf den Marsch von Etam $(13,20)$ zum Sirbonischen See $(14,2)$ vorausgeblickt, sondern v. a. auch ein Durchzug durch das Meer (gemäß P) bei Nacht (gemäß "J") ermöglicht. 14,19f. ist dann in der vorliegenden Textgestalt so zu verstehen, daß sich die Wolkensäule bei Einbruch der Nacht in eine Feuersäule verwandelte und damit Israeliten wie Ägyptern die für die folgenden Aktionen nötige visuelle Orientierung ermöglichte. ${ }^{31}$

Damit ergab sich nun aber in 14,24 die neue Schwierigkeit, daß eine "Feuersäule« allein jetzt nicht mehr (wie bei "J«) zur "Verwirrung « des ägyptischen Heeres ausreichte - war es dieser Feuersäule doch bisher anstandslos ins Meer gefolgt! R löste dieses Problem, indem sie aus der in 14,24 vorgefundenen "Feuersäule " "eine Feuer- und Wolkensäule" machte. Dabei scheint nicht an das "gewöhnliche" Phänomen des Übergangs zwischen den beiden "Säulen «-Erscheinungen von 13,21 f. gedacht zu sein, das in 14,19f. im Blick war (vgl. Ex. 40,38; Num 9,15), sondern an eine außergewöhnliche Gleichzeitigkeit beider Erscheinungen vor dem Übergang von der Nacht zum Tag. ${ }^{32}$ Diese "Feuer- und Wolkensäule»

30 Zum Problem der "Wolken"- und "Feuersäule(n)" in Ex $13+14$ vgl. W. Groß, Die Wolkensäule und die Feuersäule in Ex 13+14, in: Biblische Theologie und gesellschaftlicher Wandel, FS N. Lohfink, 1993, 142-165. Nach Groß wäre die "Feuersäule" in $13,21 \mathrm{f}$. und 14,24 redaktionell eingefügt worden (152 f.), während im "vor-P « Textbestand nur von einer "Wolkensäule " die Rede war ( $149 \mathrm{ff}$.). Groß muß dann allerdings annehmen, daß in "vor-P " die "Wolkensäule" in 14,24* determiniert war und der Artikel bei ihrer redaktionellen Ausgestaltung zu einer (idet.) "Feuer- und Wolkensäule" verlorenging ( $147 \mathrm{ff}$.). Dann läßt sich aber die Indetermination der "Feuer- und Wolkensäule" in 14,24 - nach Groß (147) die "stärkste" literarkritisch relevante "Spannung " in Ex 13+14! - letztlich doch wieder nur recht unbefriedigend als redaktionelles "Versehen " erklären.

31 Vgl. V (et erat nubes tenebrosa et inluminans noctem). Demgegenüber rechnen $\sigma^{\prime}, \mathrm{S}$ und $\mathrm{T}$ mit einer gleichzeitigen Verdunkelung (für die Ägypter) und Beleuchtung (für die Israeliten), während $G$ die Aussage über die Beleuchtung in $M$ ganz eliminiert (kai dielthen hē $n y x)$. Zur textkritischen Priorität von $M$ vgl. die Hinweise bei Groß, Wolkensäule, $146 \mathrm{f}$.

32 Die "Zeit der Morgenwache" $(14,24)$ umfaßt "das letzte Drittel der in drei ,Wachen, d. h. Wachzeiten, eingeteilten Nacht " (Noth, Exodus, 92). Vom "Anbruch des Morgens « ist erst in 14,27 die Rede. 
verwirrt nun nicht nur die Ägypter; sie bietet offenbar auch - anders als die "Feuersäule" von 13,21 f. (und 14,19f.) - Ägyptern wie Israeliten im Fortgang des Geschehens trotz "Anbruch des Morgens « $(14,27)$ nur noch sehr eingeschränkte Orientierungsmöglichkeiten. So wird verständlich, daß die Ägypter dem zurückströmenden Meer entgegenfliehen $(14,27)$ und die Israeliten offenbar erst bei hellichtem Tage ihrer Rettung gewahr werden $(14,30)$.

Neben der "Beleuchtung" des Meerwunders stellte auch die Bewegung der daran beteiligten Gruppen $\mathrm{R}$ vor Probleme. Während nämlich nach P Israeliten wie Ägypter sich ab 14,15 in Bewegung befinden, verharrten nach " J " die Israeliten die ganze Nacht über und die Ägypter bis zu ihrer "Verwirrung" durch Jahwe $\left(14,24^{*}\right)$ in Ruhe $\left(14,20^{*}\right)$. R gleicht diese Divergenz aus, indem sie in 14,19 und 25 an entscheidenden Stellen des Geschehens Aussagen einfügt, die eine Bewegung beider Gruppen voraussetzen. So interpretiert 14,19a: "Da brach der Gottes-Engel auf, der vor dem Heerlager Israels ging, und ging hinter ihnen ", die in V. 19b-20a folgende Aussage über den Positionswechsel der Wolkensäule, die sich in " J " zwischen die (ruhenden) Heerlager Israels und Ägyptens "stellte", als einen Wechsel ihrer Position bezüglich beider Heerlager in Bewegung. Daß Israeliten und Ägypter einander "nicht näher kamen " (V. 20b), ist im vorliegenden Text dementsprechend so zu verstehen, daß beide mit konstantem Abstand weitergezogen sind. $14,25 \mathrm{a}^{33}$ unterstreicht dann nochmals, daß sich die Ägypter zum Zeitpunkt ihrer "Verwirrung " durch Jahwe (V. 24) in Bewegung hinter Israel her befanden. Zudem macht diese Ergänzung verständlich, warum die Ägypter dem zurückströmenden Meer nicht entkommen konnten (V. 27 f.). Die Aufforderung Moses an Israel, "stehen zu bleiben " und "sich still zu verhalten " $(14,13 \mathrm{f}$.) wird im vorliegenden Textzusammenhang durch V. $15 \mathrm{ff}$. neu interpretiert: Sie zielt nicht auf eine völlige Passivität des Volkes, sondern darauf, daß es genau das tut, was Jahwe durch Mose anordnet, und auf eigene Initiativen zu seiner Rettung verzichtet.

Weitere redaktionelle Zusätze, die der Überbrückung von Divergenzen zwischen den vorgegebenen Quellen dienen, sind in 14,5-9 zu erkennen. V. 5b stellt einen Ausgleich her zwischen der "jahwistischen" Version des Exodus als Flucht der "beurlaubten « Israeliten und der priesterschriftlichen Darstellung einer offiziellen "Entlassung " aus dem Dienstverhältnis. Auslöser für die Verfolgung der Israeliten durch die

$33 \mathrm{Zu}$ den Interpretationsproblemen dieses Halbverses vgl. die Kommentare. Schreibt man ihn $\mathrm{R}$ zu, kann $M$ beibehalten werden: Jahwe ließ die Räder der ägyptischen Wagen "abweichen " (swr hi.) - nämlich vom trockenen Weg durch das Meer - und lenkte sie in "Schwierigkeiten " $(k b d w t)$ - wobei wohl an die morastigen Ränder dieses Weges gedacht ist. 
Ägypter ist im nun vorliegenden Text nicht mehr die Nachricht über die Flucht der Israeliten (V 5a), sondern die Reue über ihre Entlassung ( $m b$ $z$ 't 'śynw, vgl. V. 11: $m b$ z't 'śyt; zur Sache vgl. Jer 34,8ff.). Die "Diener" Pharaos sind dabei wohl im Blick auf die Plagenerzählungen ergänzt (vgl. auch 5,21), ${ }^{34}$ während die Rede vom »Herzen « Pharaos sich am unmittelbaren priesterschriftlichen Kontext orientiert (vgl. V. 4.8.17). In V. 8 gleicht $m$ lk mșrym neben dem priesterschriftlich vorgegebenen $p r^{\circ} h$ die terminologische Differenz zwischen "J" (14,5*: mlk mṣrym) und P $\left(p r^{c} h\right)$ aus. Während $\mathrm{P}$ in V. 8-10* entsprechend der Ankündigung in V. 4 von der Verfolgung der Israeliten durch Pharao spricht, ist in "J" (V. 5-10*) von einer Verfolgung durch den König von Ägypten und sein Volk die Rede. Durch ihre Zusätze in V. $8 \mathrm{f}$. verbindet $\mathrm{R}$ die singularischen Aussagen von P mit den pluralischen Formulierungen aus "J «. Die Näherbestimmung des ägyptischen Heeres in V. 9 stellt ebenso wie V. 7 klar, daß es sich bei den "Wagen " und dem "(Kriegs-)Volk ", das der König von Ägypten nach V. 6 ("J") mit sich nimmt, um »das ganze Heer" Pharaos handelt (so P in 14,4.17.28, vgl. 23).

(2) Konzeptionell entwickelt die Redaktion aus der "jahwistischen" Version des Meerwunders als Rettungswunder für Israel und seiner priesterschriftlichen (Neu-)Fassung als Erweiswunder für Ägypten ein neues Verständnis des Meerwunders als Erweiswunder für Israel. Dies wird bereits erkennbar an der redaktionellen Einleitung in 13,17 f. Indem sie die Aufforderung Jahwes an Israel, "umzukehren" (14,2: P), neu interpretiert, modifiziert sie zugleich die priesterschriftliche Konzeption des Meerwunders: Es ist nicht ausschließlich durch das Interesse Jahwes motiviert, sich an Pharao vor den Ägyptern zu verherrlichen, sondern auch und in erster Linie durch Jahwes Rücksichtnahme auf Israel. In dieser Rücksichtnahme erspart Jahwe seinem Volk nicht einfach kriegerische Auseinandersetzungen (auf die es durchaus vorbereitet ist: $13,18 \mathrm{~b} !^{35}$ ). Vielmehr "inszeniert " er (ganz im Sinne von P) das Meerwunder, das nun aber nicht primär der Rettung Israels von den Ägyptern dient (wie bei "J") oder der Selbstverherrlichung Jahwes an Ägypten (wie bei P), sondern der Einübung Israels in das Durchstehen kriegerischer Auseinandersetzungen im Vertrauen auf Jahwe und Mose (vgl. Jdc 3,1 f.). Auf dieser Linie liegen auch die redaktionellen Ergänzungen in 14,11 f. und 31. In 14,11 f. geschieht ja zunächst genau das, was Jahwe nach 13,17 f. vermeiden wollte: die Israeliten »bereuen " den Exodus, den sie "mit erhobener Hand «, d. h. »in zuversichtlicher Selbstsicherheit « 36 (14,8b) un-

34 Entsprechendes scheint für den "Stab" Moses in 14,16 zu gelten, vgl. 7,19f.; 9,22 f.; $10,12 \mathrm{f}$. und Schmidt, Studien, $19 \mathrm{f}$.

$35 \mathrm{Zu}$ ḥmš I qal Ptz. pass. s. die Lexika; vgl. Jos 1,14; 4,12; Jdc 7,11 sowie Num 32,17 (korr.).

36 Noth, Exodus, 89; vgl. Num 15,30. 
ternommen haben. Das folgende Meerwunder bewirkt dann einen Sinneswandel Israels und führt es zum "Glauben an Jahwe und an Mose, seinen Diener « $(14,31)$.

Zeigt sich hier eine hohe Wertschätzung Moses, erscheint sein Status im vorliegenden Text im Vergleich zur P-Version des Meerwunders doch deutlich reduziert: Die Israeliten werden bei ihrem Auszug aus Ägypten nicht allein durch das Wort Moses von Jahwe geführt, sondern auch durch die für alle sichtbare Wolken- bzw. Feuersäule $(13,21 \mathrm{f}$.) sowie den Gottesengel $(14,19)$, die ihnen voranziehen. Damit erhält das Volk ein höheres Maß an "Gott-Unmittelbarkeit " als in P - ohne daß die Rolle Moses als Anführer damit negiert würde. Relativiert wird auch der Anteil Moses an der Durchführung des Meerwunders in 14,15 ff.: Nachdem Mose seine Hand ausgestreckt hat, läßt Jahwe das Meer durch einen Ostwind abfließen: dadurch wird es gespalten. Anders als in $\mathrm{P}$ bewirkt Mose demnach im vorliegenden Text nicht die Spaltung des Meeres, sondern zeigt an, was Jabwe tut. ${ }^{37}$

(3) 14,31 weist mit den Stichworten "(Jahwe) fürchten «, "glauben " und "sehen" auf Aussagen im vorhergehenden Text zurück, die für die vorliegende Komposition von Ex 1-14 (15) von Bedeutung sind. Einen ersten Einschnitt markiert hier 2,24f. (P!), wo erstmals davon die Rede ist, daß Gott die Notlage Israels wahrnimmt (»hört " und "sieht «). ${ }^{38}$ Für die Zeit davor zeigt die "Gottes-Furcht " der Hebammen in 1,15-21 eine adäquate Verhaltensweise auf. ${ }^{39}$ Nach der Beauftragung Moses in Ex $3 \mathrm{f}$. kommt es für die Israeliten darauf an, auf ihn zu "hören « und ihm zu "glauben", daß ihm Jahwe "erschienen " ist $(4,1-9)$. Das tun sie zunächst auch $(4,31)$. Doch der Widerstand Pharaos gegen das Auszugsbe-

37 In der Abfolge des jetzt vorliegenden Textes in 14,1 ff. wird auch die priesterschriftliche Konzeption einer direkten Steuerung des Geschehens durch Jahwe vermittels seiner Beeinflussung des "Herzens" Pharaos etwas relativiert: Jahwe kündigt an, daß er das Herz Pharaos verhärten wird (V. 4). Das Herz Pharaos verändert sich (V. 5). Jahwe macht sein Herz hart (V. 8). Diese Abfolge läßt sich - im Sinne der priesterschriftlichen Konzeption - so verstehen, daß die "Veränderung " des Herzens Pharaos bereits Teil seiner von Jahwe bewirkten "Verhärtung " ist. V. 4+8 und V. 5 würden dann dasselbe Geschehen in ("theologischer") "Innen «- und ("empirischer ") "Außen «-Perspektive beschreiben. Der vorliegende Textablauf läßt sich aber auch so interpretieren, daß Jahwe das Herz Pharaos, das sich selbst "verändert" hat (V. 5), dann "hart macht", d. h. seine eigene Tendenz verfestigt. Damit würde der vorliegende Text gegenüber der streng "deterministischen " Konzeption der Priesterschrift die menschliche "Willensfreiheit" wenigstens als Problem (wieder) ins Spiel bringen.

38 Vgl. auch 3,7.9. Von einem "Hilfeschrei « $\left(s^{\prime} q h\right)$ der Israeliten, auf den sich Jahwe hier bezieht, berichtet im vorhergehenden Kontext nur 2,23 (wyz $q w$ [P]).

39 Vgl. auch die Diener Pharaos, die "das Wort Jahwes fürchteten « in 9,20 (aber auch V. 30). 
gehren führt sogleich zum Widerstand Israels gegen Mose $(5,21)$ : die Israeliten "hören" nicht (mehr) auf ihn (6,9: P). Erst die Wahrnehmung der göttlichen Machterweise an Ägypten ("Plagen«) bis hin zur Tötung der Erstgeburt führt wieder zu einer gewissen Bereitschaft Israels, die Anweisungen Moses zu befolgen (12,27 f., vgl. 4,31), die aber wiederum rasch durch äußere Widerstände in Frage gestellt wird $\left(14,11 \mathrm{f} .{ }^{40}\right)$. In 14,31 ist dann das "Vertrauensverhältnis « zwischen Israel und Mose wiederhergestellt (und wird in Kap. 15 hymnisch bekräftigt). Die ab 15,22 folgenden Erzählungen zeigen allerdings, daß es nicht von langer Dauer ist. Diese " 'Glaubens«-Thematik « scheint über Ex 1-14 (15) hinaus für die Redaktion des Pentateuch von Bedeutung zu sein. ${ }^{41}$ Auf einen noch weiträumigeren literarischen Horizont könnte II Reg 17,14 deuten (vgl. auch I Sam 12,16.18).

Literarische Zusammenhänge über den Pentateuch hinaus setzen jedenfalls Ex 13,19 und 14,19a voraus: Von der Bestattung der Gebeine Josephs (13,19, mit Rückbezug auf Gen 50,25) berichtet Jos 24,32 (mit Rückbezug auf Gen 33,19). Und der "Gottesengel " von Ex 14,19a begleitet Israel auf seinem Zug ins verheißene Land bis Jdc 2,1-4 (vgl. Ex $23,20.23 ; 32,34 ; 33,2$; Num 20,16; 22,22 ff.). ${ }^{42}$ Aber auch die Erwähnung des "Philister-Landes " 43 in 13,17 weist voraus auf die kriegerischen Auseinandersetzungen zwischen Israel und den Philistern in Jdc-Reg. ${ }^{44}$ Durch diese Querbezüge scheint $\mathrm{R}$ die Ereignisse vom Auszug aus Ägypten $(E x)$ bis zur Einnahme des verheißenen Landes (Jos/Jdc 2) zu einer "Epoche" der Geschichte Israels zusammenzubinden, an deren Beginn

40 Eine "Rede" der Israeliten zu Mose "in Ägypten", auf die sie in 14,12 verweisen, findet sich in dieser Form im vorhergehenden Text des Exodus-Buches nicht. Mögliche Bezugspunkte für 14,12 sind 5,21 ("J"?) und 6,9 (P!).

41 Vgl. H.-C. Schmitt, Redaktion des Pentateuch im Geiste der Prophetie, VT 32 (1982), 170-189. Vgl. Gen 15,6; Ex 19,9; Num 14,11; 20,12; Dtn 1,32; 9,23 ('mn hi.); Num 12,7; Dtn 7,9 ('mn ni.).

42 "Gottes-« bzw. "Jahwe-Engel « begegnen zuvor neben Ex 3,12 in Gen 16; 19; 21 f.; 24; 28; 31 f.; 48, danach in Jdc 5; 6; 13; II Sam 24; I Reg 13; 19; II Reg 1; 19. - Blum, Studien, $363 \mathrm{ff}$., rechnet Ex 13,19 zu einer nach-priesterlichen "Jos-24-Bearbeitung ", 14,19a hingegen zu einer vor-priesterlichen "Mal'ak-Bearbeitung “; vgl. Levin, Jahwist, 340 (zu 13,19: nach-endredaktionell) und 345 (zu 14,19a: nachjahwistische Ergänzung). Die hier vorgeschlagene Schichtung von Ex 13+14 würde demgegenüber einen späteren Ansatz von 14,19a nahelegen; vgl. auch Groß, Wolkensäule, 150 Anm. 30; H.-C. Schmitt, Die Suche nach der Identität des Jahweglaubens im nachexilischen Israel, in: J. Mehlhausen (Hg.), Pluralismus und Identität, 1995, 259-278, $261 \mathrm{f}$.

$43 \mathrm{Zu}$ "Philistern « im Pentateuch vgl. noch Gen 10,14; 21,32.34; 26,1.8.14 f.18; Ex 23,31.

44 Sie beginnen in Jdc 3,31 damit, daß Schamgar 600 Philister mit dem Ochsenstecken schlägt, vgl. die 600 auserlesenen Wagen in Ex 14,7 (R). Die Zahl 600 begegnet in Jdc-Reg dann öfter als Anzahl von Kriegern. Auch von šlyšym (Ex 14,7) ist nach Ex 15,4 dann erst wieder in Sam-Reg die Rede (erstmals II Sam 23,8). 
das Meerwunder als erster und paradigmatischer "Jahwe-Krieg " steht. ${ }^{45}$ Eben diese "Epoche" hat auch Ex 15,1-18 im Blick.

Wolken- und Feuersäule als ständige Begleiter Israels ab dem Exodus $(13,21$ f.) begegnen im Pentateuch nur noch in Num 14,14 (vgl. Neh 9,12.19). An zwei Stellen ist von "Wolke" und "Feuer " in entsprechender Funktion die Rede (Num 9,15 f.; Dtn 1,33; vgl. Ex 40,36 ff.; Num 10,34 [nur "Wolke«] sowie Ps 78,14; 105,39). Ex 33,9 f.; Num 12,5 und Dtn 31,15 sprechen von der Wolkensäule im Zusammenhang mit der Erscheinung Jahwes am bzw. im Zeltheiligtum (vgl. Ex 40,34 f. sowie Ps 99,7). Daneben begegnen "Feuer" und "Wolke" im Rahmen der Sinai- bzw. Horeb-Theophanie (Ex 19,16.18; 24,15-18; Dtn 4,11; 5,22). ${ }^{46}$ In diesem Kontext ${ }^{47}$ könnte in der Verbindung von "Wolken «- und "Feuersäule « in Ex 13,21 f.; 14,24 das Interesse an einer Verbindung der "kultischtheophanen" Präsenz Jahwes ${ }^{48}$ mit seinem "('heils--)geschichtlichen " Wirken im Exodus-Geschehen zum Ausdruck kommen, wie es auch in Ex 15,1-18 erkennbar wird.

\section{IV}

Trifft die hier vorgeschlagene Rekonstruktion zu, zeigt die Redaktion der Meerwundererzählung einen gleichermaßen "konservativen « wie "innovativen « Umgang mit den ihr vorliegenden Quellen: Diese werden allem Anschein nach vollständig und im Wortlaut bewahrt, zugleich aber auch neu interpretiert. Dabei ist die Redaktion deutlich auf einen Ausgleich der Divergenzen zwischen ihren Quellenvorgaben bedacht: ${ }^{49}$ Im Rahmen des von $\mathrm{R}$ entwickelten Geschehensablaufs werden diese

45 Bereits der "jahwistische " Grundbestand von Ex 13+14 steht den "Jahwekriegserzählungen " in Jos ff traditionsgeschichtlich nahe, vgl. F. Stolz, Jahwes und Israels Kriege, AThANT 60, 1972, 94 ff.; H. H. Schmid, Der sogenannte Jahwist, 1976, 54 ff.; Van Seters, The Life of Moses, $134 \mathrm{ff}$. Erst $\mathrm{R}$ setzt aber deutlich einen literarischen Zusammenhang zwischen Ex 13+14 und diesen Erzählungen voraus.

46 Vgl. Ps 97,2 f.; Ez 1,4 sowie "Wolke" und "Feuer " als Zeichen der "eschatologischen" Präsenz Jahwes auf dem Zion in Jes 4,5.

47 Zur redaktionsgeschichtlichen Einordnung dieser Texte vgl. die Hinweise bei Groß, Wolkensäule, $153 \mathrm{ff}$.

48 Die Präsenz der "Herrlichkeit" (kbwd) Jahwes kann sowohl mit der "Wolke" (Ex 16,10; 24,15 f.; 40,34 f.; Num 17,7) als auch mit dem "Feuer " (Ex 24,17; Lev 29,23 f.; vgl. [ohne kbwd] Lev 10,2; Num 11,1 f.; 16,35) verbunden werden; vgl. auch Ez 1,27 f.; $8,2-4 ; 10,2-6$.

49 Vgl. zu dieser redaktionellen Tendenz im Pentateuch auch Schmitt, Suche (in kritischer Auseinandersetzung mit Blum, Studien, $221 \mathrm{ff} .333 \mathrm{ff}$., und F. Crüsemann, Die Tora, 1992, $381 \mathrm{ff} .407)$ und ders., Tradition der Prophetenbücher in den Schichten der Plagenerzählung, in: Prophet und Prophetenbuch, FS O. Kaiser, BZAW 185, 1989, 196-216. 
Divergenzen $\mathrm{zu}$ miteinander vereinbaren und einander ergänzenden Aspekten eines "historischen Ereignisses «. Die literarischen und "geschichtlichen " Horizonte, in die R das Meerwunder einstellt, deuten darauf hin, daß die Redaktion der Meerwundererzählung nicht nur als Pentateuch-Redaktion anzusprechen ist, sondern als Redaktion eines GenII Reg umfassenden Geschichtswerk (mit Fortsetzung in Jes-Mal?). ${ }^{50}$

\section{Anhang:}

Exodus 13,17-14,31 (»Jahwist«-Priesterschrift-Redaktion)

13,17 Als Pharao das Volk entlassen hat(te), hat(te) Gott sie nicht den Weg zum Philister-Land geführt, obwohl es der nächste gewesen wäre. Denn Gott hat(te) gedacht: Damit das Volk es nicht bereut, wenn sie einen Kampf sehen, und sie dann nach Ägypten zurückkehren. 18 Und Gott ließ das Volk einen Umweg machen, den Wüsten-Weg zum Schilfmeer. Die Israeliten waren (bzw. sind) aber in Kampfgruppen geordnet aus dem Land Ägypten hinaufgezogen. 19 Und Mose nahm die Gebeine Josephs mit sich. Er hatte ja die Israeliten schwören lassen: Gott wird sich gewiß euer annehmen; dann sollt ihr meine Gebeine von hier mit euch hinaufbringen.

20 Und sie brachen auf von Sukkot und lagerten in Etam, am Rand der Wüste. 21 Jahwe aber ging vor ihnen her, tagsüber in einer Wolkensäule, um sie den Weg zu führen, und nachts in einer Feuersäule, um ihnen zu leuchten, damit sie tagsüber und nachts gehen konnten. 22 Nicht wich die Wolkensäule tagsüber und die Feuersäule nachts von der Spitze des Volkes.

14,1 Dann redete Jahwe zu Mose: 2 Rede zu den Israeliten, daß sie umkehren und vor Pi-Hachirot lagern sollen, zwischen Migdol und dem Meer, vor Baal-Zephon. Ihm gegenüber sollt ihr am Meer lagern. 3 Pharao aber wird von den Israeliten denken: Sie irren im Land umher; die Wüste hat sie eingeschlossen. 4 Und ich werde das Herz Pharaos hart machen, und er wird sie verfolgen. Dann will ich mich an Pharao und an seinem ganzen Heer verherrlichen, und die Ägypter werden erkennen, daß ich Jahwe bin. Und sie taten so.

5 Da wurde dem König von Ägypten gemeldet, daß das Volk geflohen war. Da veränderte sich das Herz Pharaos und seiner Diener in Richtung auf das Volk, und sie sagten: Was haben wir da getan, daß wir Israel aus unserem Dienst entlassen haben! 6 Und er ließ seine Wagen anspannen und nabm sein Volk mit sich. 7 Und er nahm 600 auserlesene Wagen

50 Vgl. Schmitt, Suche, 262. 277 f.; O. Kaiser, Der Gott des Alten Testaments. Theologie des AT 1, 1993, $157 \mathrm{ff}$. 
und alle Wagen Ägyptens. Und auf einem jeden waren hervorragende Kämpfer. $8 \mathrm{Da}$ machte Jahwe das Herz Pharaos, des Königs von Ägypten, hart. Und er verfolgte die Israeliten. Die Israeliten aber zogen mit erhobener Hand aus. 9 Und die Ägypter verfolgten sie, und sie erreichten sie, als sie am Meer lagerten, alle Wagen-Pferde Pharaos und seine Reiter und sein Heer, bei Pi-Hachirot, vor Baal-Zephon. 10 Und Pharao war nahe herangekommen. Da hoben die Israeliten ibre Augen, und siehe: die Ägypter brachen auf hinter ihnen her. Und sie fürchteten sich sehr. Da schrieen die Israeliten zu Jahwe. 11 Und sie sagten zu Mose: Hast du uns etwa, weil es keine Gräber in Ägypten gab, herausgeholt, damit wir in der Wüste sterben? Was hast du uns da angetan, daß du uns aus Ägypten herausgeführt hast? 12 War nicht das unsere Rede, die wir zu dir in Ägypten redeten: Laß uns, daß wir den Ägyptern dienen! Denn es ist besser für uns, den Ägyptern zu dienen, als in der Wüste zu sterben! 13 Da sagte Mose zu dem Volk: Fürchtet euch nicht! Bleibt stehen und seht die Hilfe Jahwes an, die er euch heute erweisen wird! Denn wie ihr die Ägypter heute gesehen habt, werdet ihr sie auf fernste Zeit niemals wieder sehen. 14 Jahwe wird für euch kämpfen, ihr aber werdet euch still verhalten!

15 Da sagte Jahwe zu Mose: Was schreist du zu mir? Rede zu den Israeliten, daß sie aufbrechen sollen! 16 Du aber hebe deinen Stab hoch und strecke deine Hand über das Meer aus und spalte es, daß die Israeliten auf dem Trockenen mitten ins Meer hineingehen können. 17 Ich aber, siehe, ich mache das Herz der Ägypter hart, daß sie hinter ihnen hineingehen, und daß ich mich verherrliche an Pharao und an seinem ganzen Heer, an seinen Wagen und an seinen Reitern. 18 Dann werden die Ägypter erkennen, daß ich Jahwe bin, wenn ich mich verherrliche an Pharao, an seinen Wagen und an seinen Reitern.

$19 \mathrm{Da}$ brach der Gottes-Engel, der vor dem Heerlager Israels ging, auf und ging hinter ihnen. Und die Wolkensäule vor ibnen brach auf und stellte sich hinter sie 20 und ging hinein zwischen das Heerlager Ägyptens und das Heerlager Israels. Da war die Wolke und die Finsternis. Dann erleuchtete sie die Nacht. Und sie näherten sich einander nicht, die ganze Nacht hindurch. $21 \mathrm{Da}$ streckte Mose seine Hand über das Meer aus. Und Jahwe ließ das Meer durch einen starken Ostwind abfließen, die ganze Nacht hindurch. Und er machte das Meer zu trockenem Land. Und das Wasser wurde gespalten. $22 \mathrm{Da}$ gingen die Israeliten auf dem Trockenen mitten ins Meer hinein. Und das Wasser war für sie eine Mauer zu ihrer Rechten und zu ihrer Linken. $23 \mathrm{Da}$ verfolgten sie die Ägypter und gingen hinter ihnen hinein, alle Pferde Pharaos, seine Wagen und seine Reiter, mitten ins Meer hinein.

24 Und zur Zeit der Morgenwache blickte Jabwe in einer Feuer- und Wolkensäule herunter auf das Heerlager Ägyptens, und er verwirrte das 
Heerlager Ägyptens. 25 Und er ließ die Räder seiner Wagen abweichen und lenkte sie in Schwierigkeiten. Da sagte Ägypten: Ich will vor Israel fliehen, denn Jahwe kämpft für sie mit Ägypten! 26 Dann sagte Jahwe zu Mose: Strecke deine Hand über das Meer aus, daß das Meer zurückkehrt über Ägypten, über seine Wagen und über seine Reiter! 27 Und Mose streckte seine Hand über das Meer aus. Da kehrte das Meer gegen Anbruch des Morgens in sein Bett zurück. Die Ägypter aber flohen ihm entgegen. So schüttelte Jahwe die Ägypter mitten ins Meer hinein. 28 Und das Meer kehrte zurück und bedeckte die Wagen und die Reiter des ganzen Heeres Pharaos, die hinter ihnen ins Meer hineingegangen waren. Kein einziger von ibnen blieb übrig.

29 Die Israeliten aber waren auf dem Trockenen mitten durchs Meer gegangen. Und das Wasser war für sie eine Mauer gewesen zu ihrer Rechten und zu ihrer Linken. 30 Und Jahwe rettete an jenem Tag Israel aus der Hand Ägyptens. Und Israel sab Ägypten tot am Ufer des Meeres. 31 Und Israel sah die große Gewalt (»Hand«), die Jahwe an Ägypten ausgeübt hatte. Da fürchtete das Volk Jahwe und glaubte an Jahwe und an Mose, seinen Diener.

Der nach Ausscheidung der priesterschriftlichen und einer "jahwistischen " Quelle in Ex 13,17-14,31 verbleibende Textbestand läßt sich interpretieren als Produkt einer Redaktion, welche die ihr vorliegenden, divergierenden Darstellungen des Meerwunders miteinander verbindet, neu interpretiert und in größere literarische Zusammenhänge einordnet. Diese Redaktion ist wohl nicht nur als Pentateuch-Redaktion anzusprechen, sondern als Redaktion eines Gen-II Reg umfassenden Geschichtswerks. 